HERAUSOEBER:

VERLAO:

Geh. San.-Rat Prof. Dr. Schwalbe

Berlin-Charlottenburg, Schlüterstrabe 53
GEORG THIEME - LEIPZIG

AntonstraBe 15
Aus der Chirurgischen Universitätsklinik in Hamburg

\section{Die Erfolge der chirurgischen Behandlung der Nephritis im Krieg und Frieden.}

Von Prof. Herm. Kümmell. ${ }^{1}$ )

M. H.! Einige Jahre vor dem Kriege habe ich in diesem Kreise über die chirurgische Behandlung der Nephritis, der letzten der in die Gruppe der "Urenzgebi_te"“ aufgenommenen Krankh.iien, berichtet. Danals war das beobachtete Krankennaterial relativ klein und die nach den stattgehabten Eingriffen verflossene Zeit noch verhältnismäBig kurz. Die Erfahrungen haben sich inzwischen vermehrt, und die "Kriegsnephritis" gab durch ihr massenhaftes Auftreten und durch"die Schwere e.ner größeren Anzahl von Fällen auch dem Chirurgen Gelegenheit, sich mit der akuten Form dieser Erkrankung eingehıend zu bcschäit.gen und hclfend da einzugreifen, wo die interne Behandlung an der Grenze ihres Könnens angelang war. Auch heute erfreut sich die operative Behandlung der Nephritis mit ihren vielgestaltigen und zum Teil noch recht wenig gekannten Bildern noch ke.ner weiten Verbreitung, auch nicht unter den Chirurgen, wie wir dies in Deutschland bei der ch.rurgischen Behandlung anderer Etkrankungen öfter bcobaclitın konnten. Ich erinnere beispielsweise an die operative Behandlung der Prostatahypertrophie, der Bauchfelltuberkulose und vor allen der Appendizitis. Nur langsam und zögernd, nach Verlauf vieler Jahre, wurden diese Erkrankungen in Deutschland der al.e n wirksamen chirtrgischen Therapie überlassen, welche sich ihrer dann schließlich mehr und mehr bemächtigte und durch die erzielten Erfolge ihre Berechtigung dazu nachwies.

Olhne auf die pathologisch-anatomische Einteilung der verschiedenen Formen der Nephritis, welche in den letzten Jahren eine wesentliche Wandlung erfahren hat, näher einzugehen, werde ich für die folgende Mitteilung eine einfachere Gruppierung wählen, welche einer wissenschaftlichen Kritik vielleicht nicht standhält, aber mir dadurch prakit.sch und übersichtlich ersch int, da $B$ sie das im Vordergrund stehende klinische Bi.d, die Symptome und Entstehungsursachen hervorhebt.

Ungezwungen wird sich die Scheidung in akute und chronische Nephritis ergeben.

\section{Akute Nephritis}

utnfaft zunächst die an akute Infekt onskrankheiten, vor allem die an Scharlach sich anschließende Nierenentzündung. Die Scharlachnephritis war es, bei welcher überhaupt zum ersten Male die Nephritis von Harrisson im Jahre 1896 operativ in Angrif genommen wurde, und zwar durch Inzis:on und Spaltung der gespannten Kapsel. Den Erfolg dieses Eingriffs erklärt er dadurch, daß das entzündlich geschwollene Organ durch die es straff umklammernde Kapsel eine intrarenale und gefährliche Behinderung seiner Zirkulation erleide. Durch Spaltung der Kapsel wird die Nierenfunktion wieder hergestellt und die gefahrdrohende Anurie beseitigt.

Die toxische Nephritis, die nicht häufig vorkommende, durch chemische Gifte hervorgerufene, schwere, akute Nierenentzündung, welche mit Anurie einhergeht und bisher erfolglos behandelt wurde, kann durch einen operativen Eingriff noch günstig beeinflußt werden. Sublimat, Kali chloricum oder ähnliche giftige Arzneimittel, welche per os dem Organismus zugeführt oder in konzentrierter Dosis angewandt wurden, gaben Veranlassung zu dieser schweren Nierenentzündung. Bei einem jungen Mädchen beispie!sweise, welches ein nicht näher festgestel'tes chemisches Gift eingenommen hatte, entstand eine schwere Anurie, welche nach 7 tägiger Dauer durch Dekapsulation beseitigt wurde. Die stark vergrößerte rechte Niere zeigte eine auffallend straffe Spannung der Kapsel und nach Spaltung und Entfernung derselben ein anfangs hochgradig anämisches, dann intensiv rotes Aussehen. Schon in den nächsten Stunden nach der Operation wurden $500 \mathrm{~g}$ Ur.n und später noch größere Mengen, bis zu dem nach 18 Stunden erfolgten Tode, entleert. Nach 7 tägiger Anurie hatte die Dekapsulation das be-

1) Nach einem im Aerztlichen Verein in Hamburg im Nor. 1919 gehaltenen Vortrag. drohliche Symptom, die Anurie, beseitigt, wenn auch Patientin an der Schwere der Allgemeinvergiftung zugrundeging.

Auch bei einer schweren toxischen Nephritis nach Sublimatvergiftung mit Anurie wurde diese durch Dekapsulation erfolgreich beseitigt.

Beider Nephrit is mit Eklampsie wurden durch die Nierendekapsulation sehr gute Erfolge erz.elt, nach Sippels Mitteilung wurden von 46 in dieser Weise operierten Eklamptischeni 30 geheilt. In vielen Publikationen ist daraut hingewiesen, daB bald nach der Operation das für diese Formen der Eklampsie gefährlichste Symptom, die Anurie, schwand, die Harnsekretion zunahm, die Krampfanfälle aufhörten, das Bewußtsein bald zurückkehrte.

Wichtiger als die erwähnten Formen der akuten Nephritis ist die $\mathrm{Nephritis}$ apostematosa in ihren verschiedenen $\mathrm{Ab}$ stufungen. Die Pyonephrose, die Eiterniere oder die Nierentuberkulose werden wir hier nicht in den Kreis unserer Betrachtungen ziehen, obwohl die Grenzen oft nicht leicht zu ziehen sind und die einzelnen Formen ineinander übeigehen können. Diese suppurative, auf hämatogenem Wege entstehende Nierenentzündung bi.'det dasjenige Gebiet der akuten Nephritis, welches durch einen chirurgischen Eingriff am günstigsten beeinflußt und zur Heilung gebracht werden kann.

Ehe wir die Frage beantworten, wann wir diese Form der akuten Nephritis aktiv angreifen sollen, möchte ich noch kurz ihre Symptome und Diagnose, gestützt auf unsere, an operierten Patienten gesammelten Erfahrungen berühren.

Es handelt sich um das Krankheitsbild, welches man mit Nephritis mit miliaren Abszessen, mit akuter suppurativer Nephritis, Nephritis apostematosa oder abscedens $u$. a. $m$. zu bezeichnen pflegt. Nicht immer ist es leicht, die ineinander übergehenden Formen vollkommen zu trennen und mit Sicherheit den Entstehungsmodus festzustellen.

Bei der infektiösen Nephritis ist die Niere mit multiplen, hirsekorn- bis stecknadelkopfgroBen AbszeBchen durchsetzt, welche meist außen auf der Rinde sichtbar sind, das Nierengewebe als solches ist entzündlich verändert und auch das Nierenbecken oft infiziert. Als charakteristisches Symptom tritt der Schmerz in der Gegend einer Niere oder in ihr selbst in den Vordergrund. Nicht immer kann man durch Palpation den Schmerz auf die Niere zurückführen oder ein vergrößertes Organ feststellen. Oft ist die erkrankte Seite mehrangespannt als andere Teile der Bauchwand, und die Muskelabwehr" ist hier oft ebenso charakteristisch, wie bei der perforierenden Appendizitis oder der Peritonitis aus anderen Gründen.

Stets bestehen neben den lokalen Nierenschmerzen die Symptome der Allgemeinerkrankung einer mehr oder weniger schweren Infektion. Uebelkeit und Erbrechen, Abgeschlagenheit und Temperatursteigerung, von Schüttelfrost begleitet. Puls beschleunigt.

Der Urin ist bei der infektiösen Nephritis infolge der Eiterkörperchen und Bakterien trüb, vorausgesetzt, da $B$ freier AbfluB aus dem erkrankten Organ nach der Blase besteht. Oft zeigt er das charakteristische Aussehen des Koliurins, die opake, leicht schillernde Färbung mit dem eigenartigen Koligeruch, wenn es sich um eine derartige Infektion handelt. Eiterkörperchen, Bakterien, rote Blutkörperchen und Zylinder sind fast stets vorhanden, letztere oft spärlich, körnige und hyaline. Albumen fehlt kaum, oft mehr vorhanden, als der Eitermenge entspricht.

Ein wichtiges diagnostisches Hilfsmittel bildet der Ureterenkatheterismus, der uns feststcllen läßt, welches die kranke Niere ist und ob die andere gesund und funktionsfähig ist. In den meisten unserer Fälle handelt es sich um eine e in seitig e Erkrankung.

In der Mehrzahl der Fälle wird die Infektion des Nierenbeckens und der Niere bei der suppurativen Nephritis auf hämatogenem Wege stattfinden. Das mit Bakterien gefüllte Blut nimmt seinen Weg durch die Niere, als das Organ, welches durch seine Funktion berufen ist, die Ausscheidung der Infektionsstoffe zu bewerkstelligen, sowie es anderseits genügend Gelegenheit gibt, die Bakterien an geeigneter Stelle in der Nierensubstanz abzulagern und dort die Bildung der kleinen Abszesse herbeizuführen. Diese kleinen metastatischen Abszesse, die in der Rinde sowohl wie im Mark auftreten und durch die Bakterien der verschiedensten Art, vor allem Bacterium coli, Streptokolken, Staphylokokken, Paratyphus und andere mehr gebildet werden, können natürlich, wenn nicht rechtzeitig ein operativer Eingriff erfolgt, konfluieren und zur Bildung 
großer AbszeBhöhlen und schließlich eines Eitersacks führcn. Oft kamen derartige Patienten in so elcndem Zustand zur Aufnahme, daß ein rasches Eingreifen notwendig war und die Feststellung der Aetiologie und der Art des Bakteriums erst aus dem entleerten Eiter stattfinden konnte.

Betrachten wir unser, die infektiöse Nephritis umfassendes Kran kenmaterial, mit Ausschluß der eigentlichen Pyonephrose, der vereiterten Niere und der Sacknierc, also speziell die Nephrit is apostematosa mit ihren multiplen Abszessen und dem getrübten Parenchym, so handelt es sich um etwa 30 Fälle im Alter von 16-60 Jahren, von denen 2 gestorben sind. Alle boten im großen und ganzen das bereits geschilderte Krankheitsbild, mehr oder weniger akutes Einsetzen der Krankheit, in den meisten Fällen hohes Fieber mit Schüttelfrost, Sclımerzen in der erkrankten Scite, Beschwerden, welche zunàchst auf cine Zystitis hinwiesen, Harndrang, Schmerzen bei der Urincntleerung und anderes mclir. Der durch den Ureterkatheter gewonnene Urin enthiclt meist reichlich Leukozyten, Zylinder und Eiweiß oft in großen Mengen, oft schr spärlicli, meistens Spuren von Blıt. In allen Fällen handelte es sich um einen einseitigen ProzeB, während die andere Niere nach den Ergebnissen der Urmuntersuchung gesund befunden wurde. Karbunkel, Typhus und Paratyphus musten in einigen Fallen als feststehende Ursachen anerkannt werden. Gonorrhoe war bei mehrerell in der Anamnese zu verzeichnen, viele Horde enthielten Bacterium coli, einige Staphylokokken und Streptokokken. Im allgemeinen sind wir früher mehr als jetzt radikal vorgegangen, sodals 17 Nephrektomien und 13 Nephrotomien und Dekapsulatıonen ausgcführt wurden.

Es ist nicht $z u$ leugnen, daB die Nephrektomie bei funktionsfähiger anderer Niere inicht nur das therapeutisch radikalste, sondern auch einfachste und für die Heilung günstigste Verfahren ist, indem bei Exstirpationen der geschlossenen, von keinem Abszeß umspülten Niere der Wundverlaut glatt und kurz ist, abgesehen davon, daB etwaige Infektionsherde, welche der andern Niere noch gefährlich werden können, vollständig entfernt werden.

Anderseits sollen wir ein so wichtigcs Organ, wie die Niere, mit allen Mitteln zu erhalten suchen und möglichst konservativ verfahren. Wir dürfen die Gefahr, dab die zurückbleibende Niere auch infektiös erkrankt und dann das Individuum von neuem dadurch aufs schwerste gefährdet wird, wie es uns ein Fall sehr drastısch vor Augen führtc, nicht unterschätzen.

Bei einer 48 jährigen Patientin wurde wegen vereiterter Niere mit Steinen die Nephrotomie vorgenommen und die Steine entfernt. Trotz freier Passage nach der Blase dauernde Harnfistel, welche sich nach verschiedenen Eingriffen nicht schloB. Da die Funktion dieser Niere wesentlich herabgesetzt war, die noch immer eiterhaltigen Urin entleerte, Nephrektomie, worauf Heilung erfolgte. Nach einigen Monaten wird Patientin im tiefsten Konna mit klemem, frcquentem Puls und hohem Fieber, urämısch und anurisch seit 3 Tagen, aufgenommen. Die vergrößerte Niere wird frcigelegt, Fcttkapsel ödematởs durchtränkt. Nach Entfernung der Capsula propria zahllose miliare Herde in, der Rinde. Spaltung der Niere, das ganze Parenchym! mit AbszeBchen durchsetzt. Nierenbecken etwas erweltert, mit trübem, eitrigem Inhalt gefüllt, Tamponade der gespaltenen Niere, Drain in das Nierenbecken eingelegt. Die Urinsekretion aus beiden Nierenhälften beginnt bald, nimmt rasch zu, Urämic schwindet, Patientin nach einigen Wochen geheilt. Der Fall zeigt uns, daß selbst bei Vorhandensein e in er Niere und schwerer infektiöser Nephritis mit Urämie noch durch Spaltung Heilung zu erzielen ist, zeigt uns anderscits aber auch die Gefahren der Nephrcktomie bei einer späteren Erkrankung der anderen Seite. Dies tritt auch bei den drei Todesfällen zutage, von denen eincr, 27 jährige Patientin, bereits das Bild einer allgemeinen Sepsis darbot und nach Entfernung der nephritischen und infizierten Niere septisch zugrundeging, während dic zuruckbleibende Nicre sich als gesund erwies. Der zweite Todesfall betraf cine Patientin mit akuter und chronischer interstitieller Nephrits mit multiplen Abszcssen, während die andere Niere gesund war und normale Funktion zeigte. Nach 3 Wochen sank der Gefrierpunkt sowie die Urinsekretion, Patientin ging urämisch zugrunde. Die Sekretion ergab Arteriosklerose und schwere Nephritis. Es ist anzunchmen, daß die arteriosklerotische Nephritis bei der Operation bestanden hat, zumal diese fast stets doppelseitig ist; jedenfalls war sie klinisch nicht nachzuweisen, und die Funktion war einwandfrei, 14 Tage blicb sic es aucl. und hatte voll und ganz die Arbeit für das fehlende Organ mit übernommen, als sic in ihrer Arbeitsfähigkeit nachlieB und vollständig versagte. Eine Nephrotomie wäre in diesem Fallc die bessere Operationsmcthode gewesen. Der dritte Todesfall entsprich ungefähr dem eben erwähnten und betrifft einen 60 jährigen Mann mit infektiöser Nephritis, von einem Nackcnkarbunkel ausgehond, bei welchem die Nephrektomie ausgeführt wurde. Auch hier wäre vielleicht durch die Nephrotomie dic während und nach der Operation funktionsfähige Nicre durch die Mitarbcit der anderen entlastet und ein günstiger Ausgang erzielt.

Daß die Nephrotomie auch ihrc Schatten in sich birgt brauche ich nicht besonders zı crwähnen, z. B. daß die Heilun ungemein verlangsamt ist, daß dic Gefahr der Nacliblutung bestcht der Eingriff kein so radikaler ist wie die Nephrektomie, daf sich eine lange Zeit bestehende Fistel ausbilden kann u. a.m. Anderseits aber ist und blcibt sie einc konscrvative Methode, welche mandochinallenden Fälen bevorzagen sollte, wo der infektiose Prozel nicht zu alusgedehnt ist, es sich nicht um eine vollständig zerstörte Niere handelt, bei welcher auch nach Ausheilung der multiplen Abszesse nicht mchr viel funk. tionierendes Gewcbe übrig bleibt. Kurz, ich würde, wenn' ich meinc Fälle und ihre Resultate ubcrblicke, zu einer vernunftigen k ons cr. vativen Therapic mit Bevorzugung der Neplirotomie mehrdas Wort reden.

In den letzten Jahren, schon vor und wahrend des Kricges, sind wir nocl konservativer in unserem aktiven Vorgehen geworden und habell die Nephrotomie, wenn sie nicht wegen Mitbeteiligung des Nierenbeckens und gröBcrer Abszesse notwendig war, durch die Dekapsulation, durch die Entfernung der Niercn. kapsel, ersetzt Durch die Enthulsung der Nierc können die oberflächlichen miliaren Abszesse frci an die Oberfläche treten, und die Bedingungen zur schncllen Hcilung sind dadurch günstig. Als Be1spiel lasse ich kurz die Krankengeschichte eines im Feldc yon mir wegen infektioser Nephritis dckapsulierten und geheilten Soldaten folgent.

M., 28 Jahre alter Infanterist, im allgemeinen gesund gewesen, seit mehreren Wochen Schmerzen in der linken Nierengegend und Fiebcr. Bei sciner Aufnahmc klagtc der Patient uber lebhafte Schmerzen, welchic sich bei der Untersuchung und leıchtem Druck lebhaft steigerten. Urin zeigt Spurcn von Eiweiß, Erythrozyten, Leukozyten und Bakterien. Temperatur bis 39 , morgens Abfall, Blutdruck 110. Wegen der anscheinenden Einseltigkeit des Leidens wird der Ureterenkatheterismus ausgeführt. Der aus der rechten Nierc entleertc Urın zeigt normale Verlälltnisse, links Leukozyten. Erythrozyten und Blutschatten. Da während der nächsten 3 Wochen der Zustand unverändert ist, dic Schmerzeıl anhalten, das Fieber naclı vorübergehendem Nachlassen mehrfach über 39 steigt, schein uns eine spontane Hcilung ausgeschlossen. Dic Diagnose wird auf infektiösc Nephritis der linken Seite gestellt und multiple Abszesse in der Niere angcnommen. Dekapsulation der Niere. Dic Kapsel ist verdickt, fest verwachisen, an einzelnen Stellen eingezogen, Nicre blutreich. Einzelne Herdchen an der Obcrfläche sichtbar. Von einer Spaltung der Nicre wird abgeschen und die verdickte Kapsel bis zum Nierenbecken entfernt. Erfolg der Operation sehr günstig, Schmerzen und Fieber schwinden, Patient ist vollkommen gesund Urin ist von Albumen und Leukozyten frei. Indigokarmin nach 10 Minuten atusgeschieden.

Daß einc akute Nephritis apostcmatosa spontan aus hcilen kann, ist möglich, aber gewiß schr selten. Mit diesen Ausnahmen sollte man cbensowenig therapeutisch rechnen, als wir

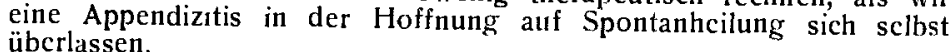

Bei kleinen Kindern habe ich wochenlang bestehende, durch Bacteritum coli und ëinmal durch Paratyphus licrvorgerufenc infektióse Nephritiden auslicilen sehen, andere starben und zeigten mcistens in beiden Nieren zahlreiche, von Bactcrium coli gebildete miliare Abszcsse. Stcht dic Diagnose der infektiösen abszedicrenden Nephritis fest, somuBdie Operation a usgeführt werden, auch dann, wenn es sich um dic sclteneren Fällc einer doppelseitigen Erkrankung handelt. Die Nephritiden mit miliaren Abszessen fuhren infolgc des großen Gefäßreicltums der Nieren und der großen Spannung, unter welcher das entzündetc Parenchym innerhalb der fibrinösen Kapsel sich befindet rasch zu einer allgemeincn Scpsis. $Z_{\text {il }}$ unterschätzen ist auch nicht die Gefahr der Infektion der andercu Niere bei eincm abwartenden Verfahren. Es kann eine toxische Nephritıs in einer gesunden Niere auftretcn und durch vaskuläre Baktericntransporte eine infektiöse Nephritis der anderen Seite entstelien. Man sollte deshalb die kranke, infizicrte Nicre sobald als möglich durch Dckapsulation oder, wenn nötig, durch $S p a l t u n g$ cntlasten, um diese zur Heilung zu ingen und die andere vor Infektion zu schützen.

Die Kriegstephritis, das gehäuftc Auftreten der Nierenerkrankungen bei Feldrugsteilnehmern, welches, im zweiten Kriegshalbjahir beginnend, in der Folge mehr und mehr zunahm, konnte nicht als einc zufällige Erkrankuung angeschen werden, sondern mußte atuch von den Erfalirenen als cine neue Kranklicitserscheinung auf-

Ansus der überrcichen Litcratur und den vielfach verschiedenen Allsichten itber dic Actiologic der Nephrritis der Kriegstcilnehmer können wir zusammenfassend im allgcucinen folgenden Schluß ziehen. Dic lange anhaltende Einwirkung ron Fcuclitigkeit und an und für kich rperlichen Ucberanstrengungen, welche ja an und für sich bei dazu Disponierten leiclıt Nierenaffcktionen hervorrufen könnell, sowie verändcrte, zum Teil cinseitige Ernährung waren dic wiclitigstenätiologisclical Motoxis der Feldncpliritis, vielleicht kombiniert mit $\mathrm{cinem}$ Der Krath hits.

Der Krankheitsverlauf und das gesamte Krankheitsbild war nach den zahlrcichen Beobachtungen zicmlich gleicl. mäbig. Eill anschcinend plötzliches Auftreten der Erkrankung, welche nach wenigen Tagen des Unbehagens dem Krieger erst zum Bewußtscin kam durch das Aluftretell von Oedemen an den
Beinen und im Gesicht.

Diese Oedcinc bildeten ein fast nie felilendes Sym. g tom in den verschiedensten Abstufungen, oft nur cine leiclite Scliwcllung der Beine, ofter ein starkes Gedunsenscin des Gesichts und Durchtränkuing der Banclidecken sowie hochgradige Schwellung des Skrotums und d's Penis. In mehrcren Fällen beobachteten wir 
stärkeren Aszites, sodaß mehrfach 3 bis 5 Liter chylöser Flüssigkeit aus dem Abdomen entleert werden mußten.

Die Atmungsorgane waren fast stets mit betroffen, Bronchitiden fehlten fast nie. Der Blutdruck war meis t e rhöht, oft bis 160 und 180 , selten bis 100 . Herzhypertrophien traten meist erst bei längerem Bestehen auf. Der Urin enthiclt fast stets Blut und zeigte die bekannte braune Farbe, zuweilen intensiv blutiges Aussehen, Zylinder ill ihren verschiedenen Formen fehlten selten, und ebenso war die Eiweißmenge meist schr reichlich, $11 / 2$ bis $3 \%$ waren keine seltene Beobachtung, in schweren Fällen stieg die Menge zu kaum meßbarer Höhe von 7 , ja sogar $10 \%$. Die Urinmenge war, dem Krankheitsbild entsprechend, oft stark vermindert bis zur hochgradigen Oligurie und Anurie. Schwere urämische Erscheinungen sahen wir öfter

In einigen Fällen kam eine vollständige A maurose, welche mit Besserung der übrigen Symptome nach einigen Tagen vollständig verschwand, zur Beobachtung. Es wird sich dabei nur um ein Oedem der Papillen gehandeit haben, Retintis albuminurica wurde in wenigen Fällen in späteren Stadien beobachtet.

Der Verlauf dieser akuten Nephritis, welche wir als eine Glomerulonephritis in den meisten Fällen ansprechen tnußten, war nach den Erfahrungen der meisten Autoren im allgemeinen günstig. Nach Ueberführung der Erkrankten in die Lazarette, bei Bettruhe, entsprechender Diät und Behandlung verschwanden meistens die akuten, oft auch die bedrohlichen urämischen Erscheinungen, um dem Stadium der Rekonvaleszenz Platz zu machen.

Rezidive, so ein erneutes Auftreten vou Blut im Urin, waren nicht selten, Albumen war in kleinen Mengen oft wochenlang vorhanden.

Wenn auch die überwiegende Mehrzahl der Kriegsnephritiden unter geeigneter Behandlung günstig verhef, so gab es doch eine nicht geringe Anzahl schwerer Fälle, die der in ternen. Therapie trotzten und bei denen man durch ein chirurgisches Vorgehen noch helfen konnte. Dic Zahl der Todesfälle be der Kriegsnephritis war immerhin nicht gering, über eine melir oder weniger grobee Anzahl Todesfälle hat jeder berichten können, welcher ein umfangreiches Nephritismaterial zu beobachten und zu behandeln gehabt hat.

In den Fällen schwerer Kriegsnephritis, sei es, daß sie sich in akutem oder subakutem Stadium befanden, in welchen die interne Therapie keine Bes. serung mehrzuerzielen imstande war, muBtedie Chi. rurgie eingreifen.

Dazu hielt ich mich für berechtigt durch meine Friedenserfahrungen. Durch Uebertragung dieser auf die Kriegsnephritis haben wir in 12 Fällen, bei welchen die üblichen internen Maßnahmen vergeblich versucht waren und bei denen Anurie und Urämie einen bedrohlichen Grad erreicht hatten, durch Dekapsulation meist nur einer Niere die Nierenfunktion in auffallend kurzer Zeit wiederherstellen und die Urämie beseitigen kònnen. Alle Patienten sind genesen, außer einem, welcher später an einer Infektion der Wunde, welche er sich durch Aufreißen des Verbandes zugezogen hatte, zugrundeging. Alle Patienten wurden frei oder fast frei von Albumen, ohne Oedem u. ä. in die Heimat entlassen. An dieser schwersten Form der Nephritis sollte keiner sterben, ohne da 3 man den aussichts. vollen Versuch der Rettung. des sonst verlorenen Lebens durch den relativ leichten Eingriff der Dekapsulation gemacht hätte. Nur wenige kurze Krankengeschichten mögen als Beispiele für den Verlauf und Erfolg des operativen Eingriffes folgen.

Ein 28 Jahre alter Soldat will früher nie krank gewesen sein. Seit 3 Wochen stark erkältet und heft ge Kopfschmerzen, seit 3 Tagen Schwellung des Gesichts. Im Feldlazarett urämische Anfälle.

Auffallend blasser Mann mit Oedemen des Gesichts und der unteren- Extremitäten. Starke Bronchitis, Kopfschmerzen, leicht benommen, Druckschmerz in der linken Nierengegend. Urin fleichwasserfarbig, $70 ; 0$ Albumen, viel Erythrozyten und hyaline Zylinder. Von neuem urämische Anfälle, welche sich trotz Venaesektion und den sonst üblichen Maßnahmen am dritten Tage zur vollständigen Bewußtlosigkeit und Anurie steigern. Im Zustand tiefsten Komas, durch Krampfanfälle unterbrochen, bei beschleunigter Respiration und beginnendem Lungenödem, wird die Dekapsulation der linken Niere in Aethernarkose ausgeführt vormittags $10 \mathrm{Uhr}$. Das exzidierte Nierenstück ergab schwere Glomerulonephritis. Abends um $8 \mathrm{Uhr}$ erwacht der Patient nach Anrufen aus seinem tiefen Schlaf und ist anscheinend klar. Urin in größeren Mengen entleert. Den anderen Tag vollständig klares Sensorium, Kopfschmerzen verschwunden, Urinsekretion an den nächsten Tagen reichlich. Ungestörte Rekonvaleszenz, nach 3 Wochen Spuren von Albumen und spärliche Zylinder. Mit gutem Allgemeinbefinden ohne subjektive Beschwerden in die Heimat entlassen.

2. T., 24 Jahre alter Infanterist, dient im 3. Jahre, war stets gesund, seit 3 Tagen kurzluftig, starke Schwellung im Gesicht und an den Füßen. Am 8. II. aufgenommen, auffallend blasser Mann, starke Oedeme im Gesicht, an Händen und Füßen, Hodensack und Bauchdecken. Ausgebreitete Bronchitis, starke Kopfschmerzen. Urin 200 Albumen, Zylinder, Urinmenge 2-3 Liter. Häufige urämische Anfälle, welche sich bis zur vollständigen Benommenheit steigern. Mehrfach wiederholte Venaesektionen und die sonst übliche Behand lung erfolglos. Am folgenden Tage bei absoluter Benommenheit, welche durclı urämische Krampfanfälle unterbrochen wird, Dekapsulation beider $\mathrm{N}$ ieren. Kapsel links stark adhärent, rechts leicht abziehbar. Beide Nieren stark vergróbert. Die Diagnose einer Glomerulonephritis wird an einem exzidierten und an Prof. Fränkel in Hamburg gesandten Stück Nierengewebe bestätigt.

Schon am folgenden Tage ist das Sensorium klarer, am 2. Tage kennt der Patient seinen Namen und seine Umgebung, und am 3. Tage ist das Sensorıum vollkommen frei Bei zunehmender Besserung stieg die Urinmenge auf $2000-2500$ bei $2 \frac{1}{2}-3$ Liter Flüssigkeitseinfuhr. Geringe Albumenmenge, einige granulierte Zylinder, keine hyalinen vorhanden. Appetit des Patıenten sehr gut, auch die Allgemeinfunktion der Nieren, deren Prüfung in Ermangelung der feineren Methoden, Kryoskopie u. a., mit Farbstofflösung, dem bewährten Indigokarmin, ausgeführt wurde, normal. Gewichtsprüfung ergibt Zunahme von 6 Pfund, Allgeneinbefinden sehr gut. (SchluB tolgt.) 